\title{
Transporter-Mediated Disposition, Clinical Pharmacokinetics and Cholestatic Potential of Glyburide and Its Primary Active Metabolites ${ }^{\mathbb{S}}$
}

\author{
Rui Li, Yi-an Bi, Anna Vildhede, Renato J. Scialis, ${ }^{1}$ Sumathy Mathialagan, Xin Yang, \\ Lisa D. Marroquin, Jian Lin, and Manthena V.S. Varma \\ Systems Modeling and Simulation, Medicine Design, Pfizer Worldwide R\&D, Cambridge, Massachusetts (R.L.); and \\ Pharmacokinetics, Dynamics and Metabolism, Medicine Design, Pfizer Worldwide R\&D, Groton, Connecticut (Y.B., A.V., R.J.S., \\ S.M., X.Y., L.D.M., J.L., M.V.S.V.)
}

Received December 30, 2016; accepted April 19, 2017

\begin{abstract}
Glyburide is widely used for the treatment of type 2 diabetes. We studied the mechanisms involved in the disposition of glyburide and its pharmacologically active hydroxy metabolites M1 and M2b and evaluated their clinical pharmacokinetics and the potential role in glyburide-induced cholestasis employing physiologically based pharmacokinetic (PBPK) modeling. Transport studies of parent and metabolites in human hepatocytes and transfected cell systems imply hepatic uptake mediated by organic anion-transporting polypeptides. Metabolites are also subjected to basolateral and biliary efflux by P-glycoprotein, breast cancer resistance protein, and multidrug resistance-associated proteins, and are substrates to renal organic anion transporter 3. A PBPK model in combination with a Bayesian approach was developed considering the identified disposition mechanisms. The model reasonably described plasma
\end{abstract}

concentration time profiles and urinary recoveries of glyburide and the metabolites, implying the role of multiple transport processes in their pharmacokinetics. Predicted free liver concentrations of the parent ( 30 -fold) and metabolites ( 4-fold) were higher than their free plasma concentrations. Finally, all three compounds showed bile salt export pump inhibition in vitro; however, significant in vivo inhibition was not apparent for any compound on the basis of a predicted unbound liver exposure-response effect model using measured in vitro IC $_{50}$ values. In conclusion, this study demonstrates the important role of multiple drug transporters in the disposition of glyburide and its active metabolites, suggesting that variability in the function of these processes may lead to pharmacokinetic variability in the parent and the metabolites, potentially translating to pharmacodynamic variability.

\section{Introduction}

Glyburide (also called glibenclamide), a second-generation sulfonylurea, is widely used for the treatment of type 2 diabetes. It is a potent stimulator of pancreatic insulin secretion and may additionally decrease

${ }^{1}$ Current affiliation: Pharmaceutical Candidate Optimization, Bristol-Myers Squibb, Princeton, New Jersey.

All authors are full-time employees of Pfizer Inc. No other potential conflicts of interest relevant to this article are reported.

https://doi.org/10.1124/dmd.116.074815.

SThis article has supplemental material available at dmd.aspetjournals.org. the resistance of muscle and liver to the action of insulin (Feldman, 1985). Glyburide is an extended clearance classification system class $1 \mathrm{~B}$ drug (Varma et al., 2015) with evidence for active hepatic uptake via organic anion transporting polypeptide (OATP) 1B1 and subsequent metabolism (Naritomi et al., 2004; Zhou et al., 2010; Varma et al., 2014). CYP2C9 is thought to be primarily responsible for the biotransformation of glyburide in vivo, with CYP3A4 playing a minor role. Several clinical studies demonstrate an association between glyburide pharmacokinetics and genetic polymorphism of CYP2C9, where the carriers of CYP2C9*3 variants show reduced clearance (Kirchheiner et al., 2002; Niemi et al., 2002; Ravindran et al., 2006). On the contrary, in vitro

\footnotetext{
ABBREVIATIONS: BCRP, breast cancer resistance protein; BSEP, bile salt export pump; $\mathrm{Cl}$, confidence interval; $C L_{\text {liver, } u \text {,act }}$, hepatic unbound active uptake clearance; $C L_{\text {liver,u,bile, }}$, hepatic unbound biliary excretion clearance; $C L_{\text {liver,u,efflux }}$, hepatic unbound basal efflux clearance; $C L_{\text {liver,u,pass, }}$ hepatic unbound passive diffusion clearance; $C L_{R}$, plasma renal clearance; $C L_{u \text {,act }}$, sandwich-cultured human hepatocyte or plated human hepatocyte unbound active uptake clearance; $C L_{u \text {,bile }}$, sandwich-cultured human hepatocyte unbound biliary excretion clearance; $C L_{u, p a s s}$ sandwich-cultured human hepatocyte or plated human hepatocyte unbound passive diffusion clearance; DDI, drug-drug interaction; $F_{a}$, fraction of drug absorbed; $F_{g}$, fraction of drug escaping gut-wall extraction; $F_{M 1}$, fraction of glyburide converted to $M 1 ; F_{M 2 b}$, fraction of glyburide converted to M2b; $f_{u, c}$, intracellular free fraction; $f_{u, p}$, fraction unbound in plasma; HBSS, Hanks' balanced salt solution; HEK, human embryonic kidney; HLM, human liver microsome; IVIVE, in vitro to in vivo extrapolation; $k_{a, G}$, absorption rate of glyburide; $K p_{u u}$, pseudo-steady-state unbound liver tissue-tounbound plasma ratio; LC-MS/MS, liquid chromatography tandem mass spectroscopy; M1, 4-trans-hydroxyglyburide; M2b, 3-cis-hydroxyglyburide; MCMC, Markov chain Monte Carlo; MDR1, multidrug resistance protein 1; MOPS, 3-(N-morpholino)propanesulfonic acid; MRP, multidrug resistance-associated protein; NTCP, sodium/taurocholate cotransporting polypeptide; OAT, organic anion transporter; OATP, organic anion transporting polypeptide; P-gp, P-glycoprotein; $\mathrm{PHH}$, plated human hepatocyte; $\mathrm{PBPK}$, physiologically based pharmacokinetic; $\mathrm{SCHH}$, sandwichcultured human hepatocyte.
} 
enzymology studies suggest CYP3A4 to be the major metabolizing enzyme, with CYP2C9 playing a minimal role (Zharikova et al., 2009; Zhou et al., 2010). Based on the in vitro studies and mechanistic modeling of clinical pharmacokinetics and drug-drug interactions (DDIs) of glyburide as a victim drug, we recently assessed the quantitative role of the transporter-enzyme (OATP1B1-CYP2C9/3A4) interplay in its hepatic clearance (Varma et al., 2014).

Glyburide is excreted as hydroxy metabolites with $50 \%$ of the dose in the urine and about $50 \%$ of the dose in the bile (Feldman, 1985). The two major circulating metabolites, 4-trans-hydroxyglyburide (M1) and 3-cis-hydroxyglyburide (M2b), were shown to have $\sim 50-75 \%$ of the hypoglycemic activity of the parent due to increased insulin secretion (Rydberg et al., 1994). These two major metabolites are rapidly cleared from the bloodstream when dosed intravenously (Rydberg et al., 1995) and may have higher activity at low concentrations with longer effect duration than the parent drug (Rydberg et al., 1997; Jonsson et al., 2001). However, the transport and metabolic processes involved in the disposition of these metabolites are not characterized.

Drug-induced cholestasis is often noted and associated with bile salt export pump (BSEP) inhibition (Rodrigues et al., 2014). Examples of drugs that are known to induce cholestasis and cholestatic or mixed hepatitis potentially via inhibition of BSEP include cyclosporine A, rifampicin, bosentan, and glyburide. Bosentan induces a dose-dependent liver injury and increased serum bile salts and alkaline phosphatase levels in a significant proportion of treated patients (Fattinger et al., 2001). Additionally, the cholestatic potency of bosentan is elevated when combined with glyburide in humans (Mylona and Cleland, 1999; Fattinger et al., 2001). As a result, this combination is contraindicated in clinical practice (bosentan product label). Bosentan and its metabolites and glyburide are known to inhibit BSEP, which is believed to be a major cause of the observed cholestatic findings (Fattinger et al., 2001). The contribution of glyburide major metabolites (M1 and $\mathrm{M} 2 \mathrm{~b}$ ) to the glyburide-induced liver injury has not been studied.

The goals of this study were 1) to evaluate the transport mechanisms involved in the hepatic and renal disposition of glyburide and its M1 and M2b metabolites, 2) to characterize the clinical pharmacokinetics of glyburide and the metabolites using a physiologically based pharmacokinetic (PBPK) model, and 3) to quantitate the hepatic exposure of the parent and metabolites and project their quantitative role in the glyburide-induced cholestasis.

\section{Materials and Methods}

\section{Chemicals and Reagents}

Glyburide and rifamycin SV were purchased from Sigma-Aldrich (St. Louis, $\mathrm{MO}$ ). M1 and M2b were obtained from Medical Isotopes (Pelham, NH). InVitroGRO-HT, CP, and HI hepatocyte media were purchased from Celsis IVT (Baltimore, MD). Cryopreserved human hepatocytes from donor HH1025 and HH1026 (Caucasian female, 59 years old) were purchased from In Vitro ADMET Laboratories, LLC (Columbia, MD). Human embryonic kidney (HEK) 293 cells stably transfected with human OATP1B1, OATP1B3, or OATP2B1 were generated at Pfizer Inc. (Sandwich, UK). HEK293 cells transfected with organic anion transporter (OAT) 1 and OAT3 were obtained from Dr. Kathleen Giacomini (University of California, San Francisco, San Francisco, CA). HEK293 cells stably transfected with NTCP were obtained from Professor Per Artursson (Uppsala University, Uppsala, Sweden). Human multidrug resistanceassociated protein (MRP) 2, MRP3, MRP4, and breast cancer resistance protein (BCRP) vesicles were obtained from Corning (Corning, NY). Human BSEP and multidrug resistance protein 1 (MDR1) vesicles were purchased from Solvo Biotechnology (Budapest, Hungary).

\section{In Vitro Transport Studies}

OATPs and OAT Substrate Assay. HEK-OATP1B1, HEK-OATP1B3, HEK-OATP2B1, HEK-OAT1, HEK-OAT2, HEK-OAT3, and HEK-mock cells were seeded at densities of 60,000-90,000 cells/well in 96-well poly-D-lysinecoated plates (OATP assays) or 300,000 cells/well in 24-well plates (OAT assays) and cultured for 48 hours. For the uptake assays, the cells were washed three times with uptake buffer [Hanks' balanced salt solution (HBSS) with 20 mM HEPES, $\mathrm{pH}$ 7.4] and then incubated with uptake buffer containing test compound at $37^{\circ} \mathrm{C}$ and $150 \mathrm{rpm}$. Cellular uptake was terminated by quickly washing the cells three or four times with ice-cold uptake buffer. The cells were then lysed with methanol containing internal standard, and the samples were quantified by liquid chromatography tandem mass spectroscopy (LC-MS/MS). The total cellular protein content was determined by using the Pierce BCA Protein Assay Kit (ThermoFisher Scientific, Waltham, MA) according to the manufacturer specifications.

MRPs, BCRP, and MDR1 Substrate Assay. M1 and M2b were evaluated for ATP-dependent transport by MRP2, MRP3, MRP4, BCRP, and MDR1 at 1 and $10 \mu \mathrm{M}$ concentrations. The assays were conducted in 96-well format using the rapid filtration technique. Briefly, $50 \mu \mathrm{g}$ of membrane vesicles were incubated with test compound for 5 minutes at $37^{\circ} \mathrm{C}$ in the presence of $5 \mathrm{mM}$ ATP or $5 \mathrm{mM}$ AMP in buffer containing $2.5 \mathrm{mM}$ glutathione, $70 \mathrm{mM} \mathrm{KCl}, 7.5 \mathrm{mM} \mathrm{MgCl}_{2}$, and $50 \mathrm{mM}$ MOPS [3-( $N$-morpholino)propanesulfonic acid] adjusted to $\mathrm{pH} 7.4$ with Tris (MRP2 and MRP3) or $250 \mathrm{mM}$ sucrose, $10 \mathrm{mM} \mathrm{MgCl} 2$, and $10 \mathrm{mM}$ Tris adjusted to $\mathrm{pH} 7.4$ with $\mathrm{HCl}$ (MRP4, BCRP, and MDR1). The transport reaction was stopped by the addition of cold stop buffer $(70 \mathrm{mM} \mathrm{KCl}$ and $40 \mathrm{mM}$ MOPS adjusted to $\mathrm{pH} 7.4$ with Tris for MRP2 and MRP3 and $100 \mathrm{mM} \mathrm{NaCl}$ in assay buffer for MRP4, BCRP, and MDR1). Samples were transferred to 96-well glass fiber filter plates, filtered, and washed four times with cold stop buffer. Accumulation of the test compound in the membrane vesicles was measured by extracting the compound with methanol containing internal standard followed by LC-MS/MS analysis.

BSEP Inhibition Assay. Glyburide, M1, and M2b were evaluated for inhibition of BSEP-mediated ATP-dependent transport of taurocholic acid using membrane vesicles. The assay was conducted in a 384-well format at 11 concentrations per compound. The rapid filtration method was used as described previously with some modifications (Dawson et al., 2012). Briefly, $16 \mu \mathrm{g}$ of BSEP vesicles were incubated with $2 \mu \mathrm{M}$ taurocholic acid and test compound or dimethylsulfoxide for 40 minutes at $25^{\circ} \mathrm{C}$ in buffer containing $4 \mathrm{mM}$ ATP, $100 \mathrm{mM} \mathrm{KNO}_{3}, 10 \mathrm{mM} \mathrm{Mg}\left(\mathrm{NO}_{3}\right)_{2}, 50 \mathrm{mM}$ sucrose, and $50 \mathrm{mM}$ HEPES, pH 7.4. The transport reaction was stopped by the addition of cold $0.5 \mathrm{M}$ EDTA and cold stop buffer (10 mM Tris, pH 7.4; $100 \mathrm{mM} \mathrm{KNO}_{3} ; 10 \mathrm{mM} \mathrm{Mg}\left(\mathrm{NO}_{3}\right)_{2}$; and $50 \mathrm{mM}$ sucrose). The samples were rapidly filtered and washed three times with ice-cold buffer. After the filter plate was dried, taurocholic acid was extracted from the vesicles by adding methanol and water (80:20 ratio) to the filter plate, and its concentration was measured by LC-MS/MS.

NTCP Inhibition Assay. Glyburide, M1, and M2b were evaluated for inhibition of NTCP-mediated transport of taurocholic acid at nine concentrations per compound. HEK-NTCP cells were seeded at a density of 60,000 cells/well in poly-D-lysine-coated 96-well plates and cultured for 48 hours. The cells were washed three times with uptake buffer (HBSS with $20 \mathrm{mM}$ HEPES, $\mathrm{pH}$ 7.4) and then incubated for 4 minutes with uptake buffer containing $0.4 \mu \mathrm{M}^{3} \mathrm{H}$-taurocholic acid and test compound at $37^{\circ} \mathrm{C}$ and $150 \mathrm{rpm}$. Uptake was stopped by the removal

TABLE 1

Hepatic OATP-mediated transport of glyburide and metabolites

Uptake by HEK cells stably transfected with human OATP1B1, OATP1B3, and OATP2B1, normalized to uptake by wild-type HEK cells, are presented as uptake ratios. All data represent the mean $\pm \mathrm{SD}(n=3)$.

\begin{tabular}{lrcr}
\hline \multicolumn{1}{c}{ Compound } & \multicolumn{1}{c}{ OATP1B1 } & OATP1B3 & OATP2B1 \\
\hline Glyburide $1 \mu \mathrm{M}$ & $1.9 \pm 0.2$ & $1.2^{a}$ & $1.3^{a}$ \\
Glyburide $10 \mu \mathrm{M}$ & $1.2 \pm 0.1$ & & \\
M1 $1 \mu \mathrm{M}$ & $26.1 \pm 1.8$ & $17.5 \pm 2.6$ & $15.2 \pm 0.3$ \\
M1 $10 \mu \mathrm{M}$ & $46.7 \pm 0.7$ & $35.4 \pm 3.5$ & $21.4 \pm 0.7$ \\
M2b $1 \mu \mathrm{M}$ & $28.1 \pm 0.7$ & $22.2 \pm 6.8$ & $20.2 \pm 0.3$ \\
M2b $10 \mu \mathrm{M}$ & $39.1 \pm 1.2$ & $30.8 \pm 0.8$ & $24.8 \pm 1.8$ \\
Propranolol $1 \mu \mathrm{M}^{b}$ & $1.1 \pm 0.1$ & $1.4 \pm 0.1$ & $1.3 \pm 0.1$ \\
Rosuvastatin $1 \mu \mathrm{M}^{b}$ & $55.3 \pm 1.1$ & $14.2 \pm 0.5$ & $36.2 \pm 2.9$ \\
\hline
\end{tabular}

${ }^{a}$ Taken from our previous work (Varma et al., 2014).

${ }^{b}$ Propranolol served as negative control and rosuvastatin served as positive control for all three OATPs. 
TABLE 2

Hepatic BCRP, MDR1, and MRPs mediated transport of glyburide metabolites

Uptake by inverted membrane vesicles overexpressing human BCRP, MDR1, MRP2, MRP3, and MRP4 in the presence of ATP normalized to uptake in presence of AMP are presented as transport ratios. All data represent the mean $\pm \operatorname{SD}(n=2)$.

\begin{tabular}{|c|c|c|c|c|c|}
\hline Compound & BCRP & MDR1 & MRP2 & MRP3 & MRP4 \\
\hline M1 $1 \mu \mathrm{M}$ & $7.39 \pm 0.66$ & $4.89 \pm 0.59$ & $1.17 \pm 0.13$ & $1.58 \pm 0.18$ & $2.03 \pm 0.08$ \\
\hline M1 $10 \mu \mathrm{M}$ & $3.86 \pm 0.27$ & $4.30 \pm 0.44$ & $0.99 \pm 0.11$ & $1.72 \pm 0.22$ & $2.55 \pm 0.15$ \\
\hline $\mathrm{M} 2 \mathrm{~b} 1 \mu \mathrm{M}$ & $2.89 \pm 0.31$ & $3.91 \pm 0.24$ & $0.85 \pm 0.03$ & $0.97 \pm 0.14$ & $1.05 \pm 0.04$ \\
\hline $\mathrm{M} 2 \mathrm{~b} 10 \mu \mathrm{M}$ & $2.34 \pm 0.03$ & $3.65 \pm 0.08$ & $1.15 \pm 0.16$ & $0.94 \pm 0.21$ & $1.36 \pm 0.16$ \\
\hline Estrone sulfate $1 \mu \mathrm{M}(\mathrm{BCRP})^{a}$ & $14.13 \pm 0.08$ & & & & \\
\hline$N$-methyl quinidine $1 \mu \mathrm{M}(\mathrm{hMDR} 1)^{a}$ & & $24.70 \pm 1.11$ & & & \\
\hline Leukotriene C4 $0.1 \mu \mathrm{M}(\mathrm{hMRP})^{a}$ & & & $30.8 \pm 1.68$ & $18.27 \pm 2.54$ & $22.54 \pm 0.82$ \\
\hline
\end{tabular}

hMDR1, human MDR1; hMRP, human MRP.

${ }^{a}$ Probe substrate was used as a positive control for the respective transporter.

of transport buffer followed by three washes with ice-cold buffer. The cells were lysed with $100 \mu$ l of $10 \mathrm{mM}$ Tris-HCL ( $\mathrm{pH} 7.5$ ), $75 \mathrm{mM} \mathrm{NaCl}, 125 \mathrm{mM} \mathrm{NaF}$, $2.5 \mathrm{mM}$ EDTA, and $0.5 \% \mathrm{NP} 40$, and shaken for 45 minutes at room temperature. Accumulated radioactivity was determined by mixing $50 \mu \mathrm{l}$ of cell lysate with $220 \mu \mathrm{l}$ of scintillation fluid and analyzing the samples on a PerkinElmer (Waltham, MA) MicroBeta TriLux Liquid Scintillation Counter.

Sandwich-Cultured Human Hepatocyte and Plated Human Hepatocyte Transport Assays. The sandwich-cultured human hepatocyte (SCHH) methodology was described previously (Bi et al., 2006). Briefly, cryopreserved human hepatocytes were thawed and seeded in 24-well collagen-coated plates using InVitroGRO-HT and InVitroGRO-CP media. The plates were overlaid with $0.25 \mathrm{mg} / \mathrm{ml}$ Matrigel (Corning) on the second day, and the cultures were maintained in InVitroGRO-HI medium. On day 5, the cells were preincubated for 10 minutes with or without $100 \mu \mathrm{M}$ rifamycin SV (to determine the rates of passive diffusion and total uptake, respectively) in buffer with or without $\mathrm{Ca}^{++}$(to determine biliary clearance). The reactions were terminated at specified time points by washing the cells three times with ice-cold HBSS. The cells were lysed with methanol containing internal standard, and intracellular concentrations were determined by LC-MS/MS.

The plated human hepatocyte (PHH) uptake study was conducted to determine the intracellular free fraction $\left(f_{u, c}\right)$ with a longer incubation time. The assay was conducted 6 hours after seeding (without overlaying with matrigel), as described for the SCHH assay with the exception of $\mathrm{Ca}^{++}$-free incubations.

LC-MS/MS Analysis. LC-MS/MS analysis was conducted for all in vitro samples using a SCIEX (Framingham, MA) 5500 or 6500 Triple Quadrupole Tandem Mass Spectrometer in electrospray ionization mode. Other instrumentation consisted of Shimadzu (Kyoto, Japan) LC-20AD Solvent Delivery Units (pumps) and ADDA autosampler. Liquid chromatography was performed using a Phenomenex (Torrance, CA) Kinetex C18 or Synergi Polar-RP $(30 \times 2 \mathrm{~mm})$ column, or a Sprite Echelon C18 column $(10 \times 2.1 \mathrm{~mm})$ (ANALYTICAL Sales and Serivices, Flanders, NJ). Analytes were eluted with a gradient profile starting with $0.1 \%$ formic acid in water and increasing concentration of $0.1 \%$ formic acid in acetonitrile.

TABLE 3

Renal OATs mediated transport of glyburide and metabolites

Uptake by HEK cells stably transfected with human OAT1 and OAT3, normalized to uptake by wild-type HEK cells, are presented as uptake ratios. All data represent the mean $\pm \operatorname{SD}(n=3)$.

\begin{tabular}{|c|c|c|}
\hline Compound & OAT1 & OAT3 \\
\hline Glyburide $1 \mu \mathrm{M}$ & $1.24 \pm 0.16$ & $1.25 \pm 0.10$ \\
\hline Glyburide $10 \mu \mathrm{M}$ & $1.25 \pm 0.09$ & $0.97 \pm 0.07$ \\
\hline M1 $1 \mu \mathrm{M}$ & $1.48 \pm 0.20$ & $6.22 \pm 0.04$ \\
\hline M1 $10 \mu \mathrm{M}$ & $0.37 \pm 0.02$ & $11.50 \pm 0.86$ \\
\hline $\mathrm{M} 2 \mathrm{~b} 1 \mu \mathrm{M}$ & $0.64 \pm 0.06$ & $9.25 \pm 0.54$ \\
\hline $\mathrm{M} 2 \mathrm{~b} 10 \mu \mathrm{M}$ & $0.96 \pm 0.63$ & $13.51 \pm 0.72$ \\
\hline${ }^{3} \mathrm{H}$-para-aminohippuric acid (hOAT1) ${ }^{a}$ & $74.13 \pm 5.69$ & \\
\hline${ }^{3} \mathrm{H}$-estrone sulfate $(\mathrm{hOAT} 3)^{a}$ & & $95.04 \pm 1.98$ \\
\hline
\end{tabular}

hOAT, human OAT.

${ }^{a}$ Probe substrate used as positive control for the respective transporter.
Mechanistic Modeling of Hepatocyte Uptake Studies. Mechanistic modeling of SCHH data to estimate in vitro unbound active uptake clearance $\left(C L_{u, a c t}\right)$, unbound passive diffusion clearance $\left(C L_{u, p a s s}\right)$, unbound basolateral efflux clearance $\left(C L_{u, \text { efflux }}\right)$, and unbound biliary excretion clearance $\left(C L_{u, b i l e}\right)$ of glyburide and metabolites M1 and M2b were performed as described previously (Kimoto et al., 2015). The detailed model structure is provided in the Supplemental Material. The PHH data were analyzed using the mechanistic model developed for SCHH, $C L_{u, b i l e}$ set to 0 . The $f_{u, c}$ was estimated along with other parameters during $\mathrm{PHH}$ data fitting. $C L_{u, p a s s}$ and $f_{u, c}$ were assumed to be the same for the two metabolites (configurational isomers). Parameter estimation was performed using a global optimization algorithm (differential evolution) in $\log _{10}$ space, with 95\% confidence intervals (CIs) quantified by the residual bootstrap. All models in this study were implemented in MATLAB (version 2016a; MathWorks, Natick, MA).

PBPK Modeling of Glyburide and Its Active Metabolites. A previously published PBPK model for liver transporter substrates (Li et al., 2014a) was used to model the human plasma data for glyburide and its two active metabolites. Details about the structural model are provided in the Supplemental Material. Given that the physiochemical properties and in vitro uptake characteristics of the two metabolites estimated in SCHH were reasonably close and that the clinical pharmacokinetic data of the two metabolites are also similar (Rydberg et al., 1995), we assumed that hepatic active uptake $\left(C L_{\text {liver, }, \text { act }}\right)$, passive diffusion $\left(C L_{\text {liver,u,pass }}\right)$, and biliary excretion $\left(C L_{\text {liver,u,bile }}\right)$ were similar for the two metabolites to decrease the number of fitted parameters. Biliary excretion and basolateral efflux $\left(C L_{\text {liver, }, \text { efflux }}\right)$ of glyburide, as well as further metabolism of the metabolites, were assumed to be zero based on our in vitro studies. The unbound hepatic clearance processes, the fraction of glyburide converted to M1 $\left(F_{M 1}\right.$, with the ratio between $F_{M 1}$ and $F_{M 2 b}$ fixed at 5 based on clinical observation), and the absorption rate of glyburide $\left(k_{a, G}\right)$ were initially estimated using the global optimization (i.e., differential evolution) in $\log _{10}$ space to determine one set of values that best described the pooled clinical data from six independent studies with healthy participants (Neugebauer et al., 1985; Chalk et al., 1986; Spraul et al., 1989; Rydberg et al., 1995; Niemi et al., 2001; Lilja et al., 2007). Although most data are reasonably consistent, the first-hour data reported in the studies by Spraul et al. (1989) and Rydberg et al. (1995), and Neugebauer et al. (1985) can lead to different conclusions about glyburide tissue distribution. For this reason, we removed the first-hour data reported in the studies by Spraul et al., 1989) and Rydberg et al. (1995) from fitting [assuming that intravenous infusion data (Neugebauer et al., 1985) better predicts distribution volume]. Data 10 hours postdose were not simulated to avoid large errors that may incur when digitizing these extreme low concentrations from non-log-transformed plots. The physiologic parameters were the same as described previously (Rodgers and Rowland, 2006; Li et al., 2014b).

The distributions of nine fitted parameters were estimated using a Bayesian inference where both previous knowledge about in vitro to in vivo extrapolation (IVIVE) translation (i.e., prior distribution) and clinical data for glyburide (i.e., likelihood) contributed to parameter estimates (i.e., posterior distribution). Alternatively, parameter estimation during fitting of clinical data for glyburide is constrained by our best guess about IVIVE learned from other compounds. With the "middle-out" approach described previously (Li et al., 2014b), the distributions IVIVE empirical scaling factors (for SCHH, lot $\mathrm{HH} 1025$ ) have been 
A

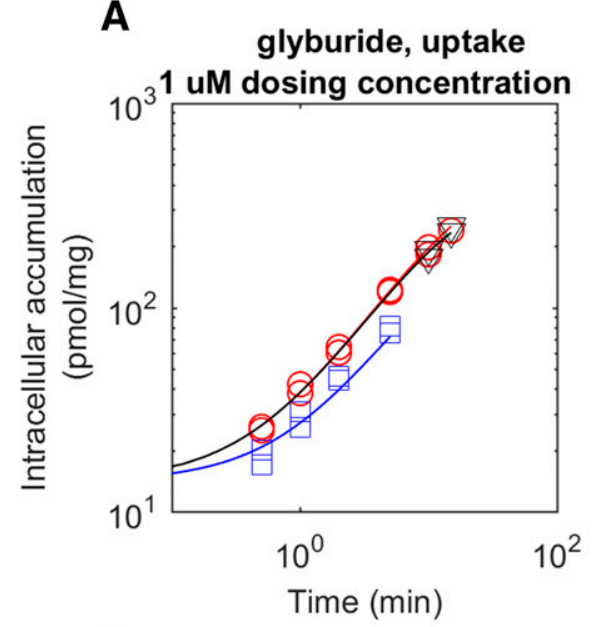

D

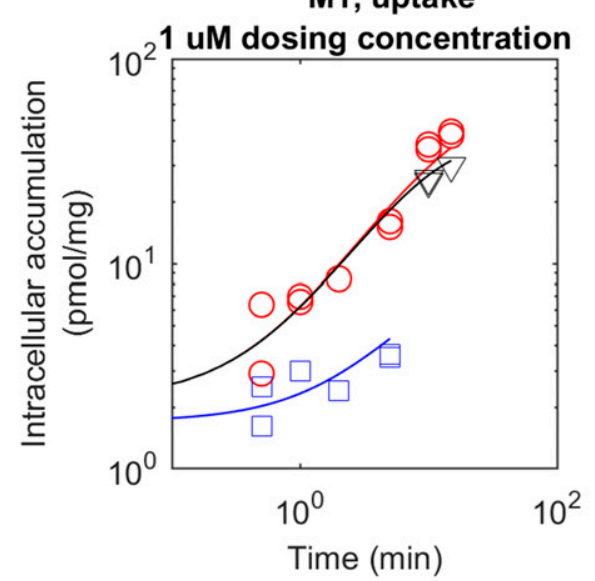

G

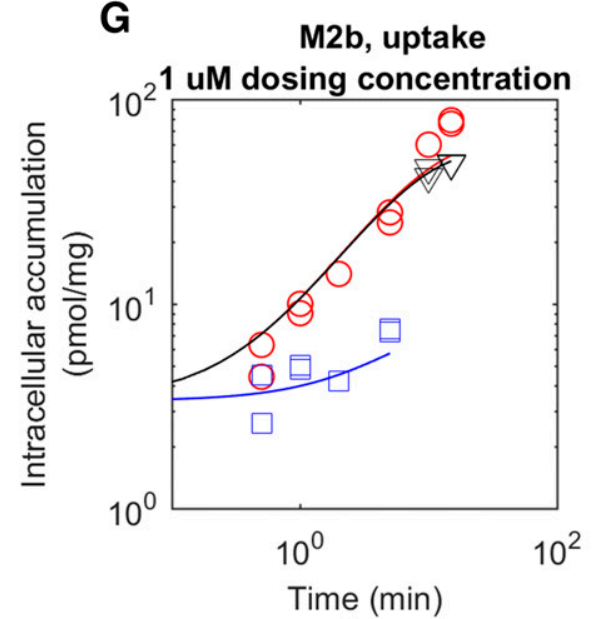

B

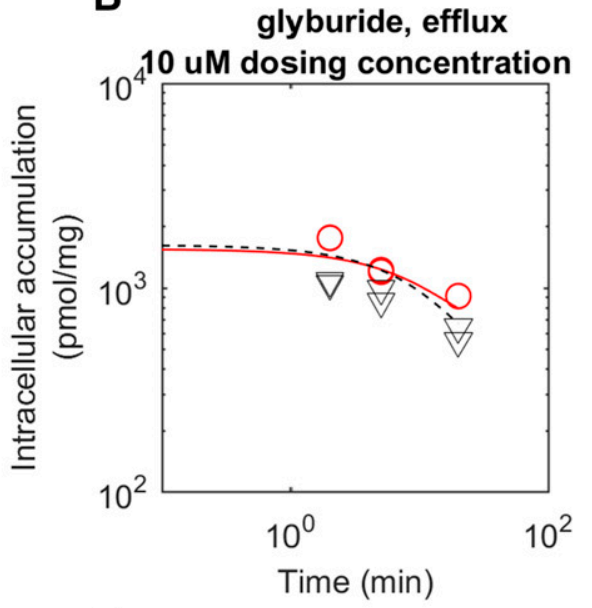

$\mathbf{E}$

M1, efflux

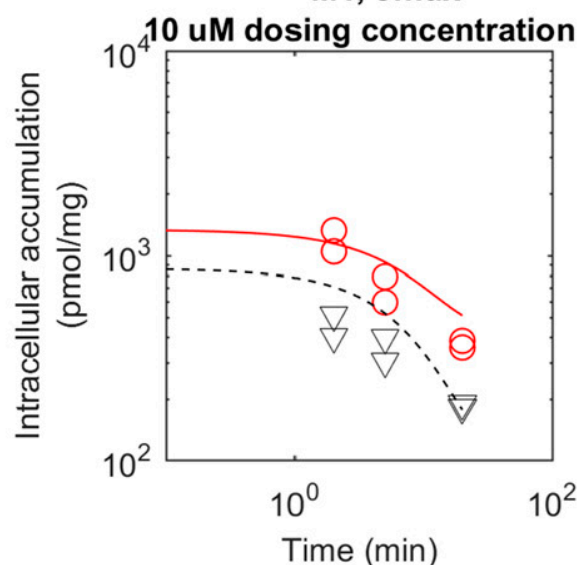

H

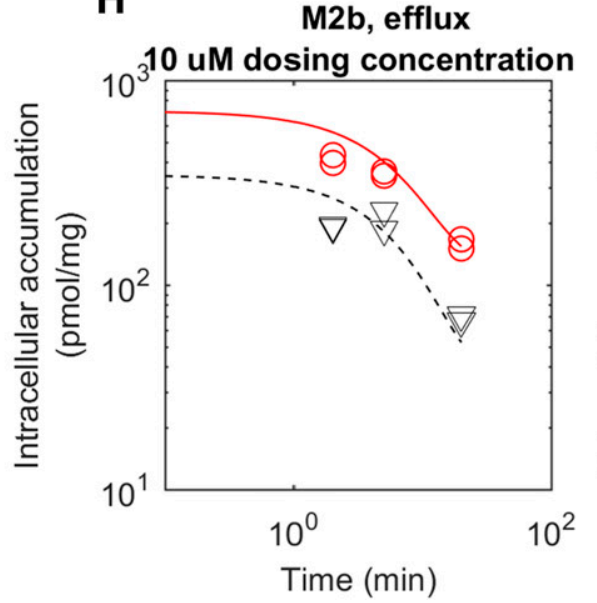



$\mathbf{F}$

M1, efflux

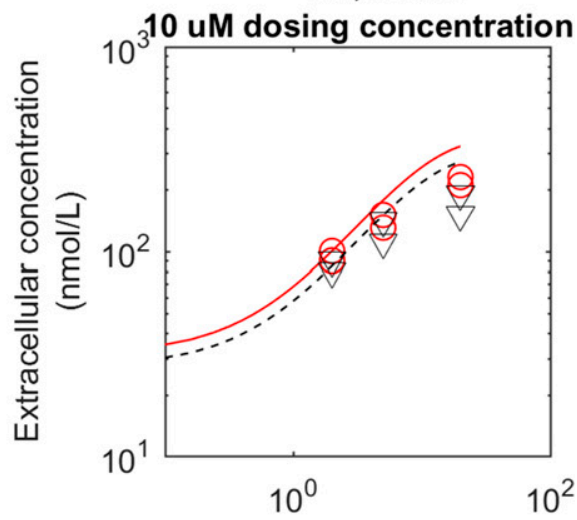

Time (min)

M2b, efflux

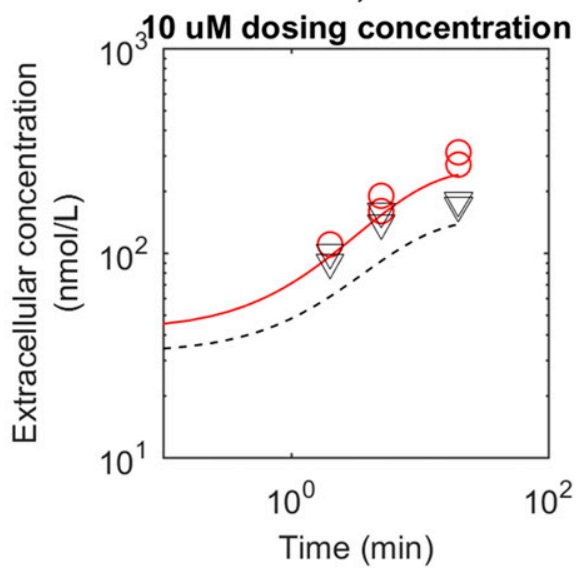

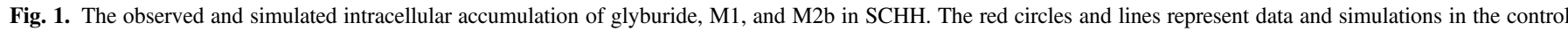

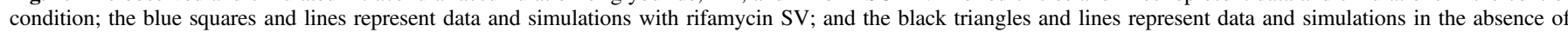

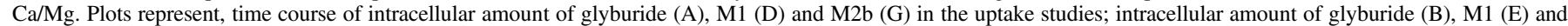
M2b (H) in the efflux studies; and extracellular amount of glyburide (B), M1 (E) and M2b (H) in the efflux studies.

estimated using six structurally different liver transporter substrates (i.e., $10^{1.52 \pm 0.31}$, $10^{-0.875 \pm 0.52}$, and $10^{-0.857 \pm 0.27}$, as means and S.D.s in $\log _{10}$ space for active uptake, passive diffusion, and metabolism; unpublished internal data). Briefly, the Bayesian approach includes three steps. In step 1, we calculated the prior distribution of $C L_{\text {liver,u,act }}, C L_{\text {liver,u,pass, }}$, and $C L_{\text {liver,u,bile }}$ as the products of the previously estimated IVIVE empirical scaling factors, the physiologic scaling factor of 120 million hepatocytes per gram of liver tissue, and the in vitro $\mathrm{SCHH}$ (or HLM) clearances (for M1 and M2b, the averaged values of the two compounds were used). For the metabolites intrinsic biliary and basolateral efflux clearances, $C L_{\text {liver, }, \text { bile, }, M}$ and $C L_{\text {liver, }, \text { efflux }, M}$, since we had no knowledge about their IVIVE, we assumed that their prior distributions were uniform and bounded by starting values divided and multiplied by 1000 , whereas $k_{a, G}$ and $F_{M}$ were upper bounded by 10 and $5 /(5+1)$, respectively. In step 2 , the likelihood is calculated as the sum of the squared error between pooled clinical data and simulations in $\log _{10}$ space. In step 3, the posterior distributions of the estimated parameters (i.e., parameter values specific for glyburide and its metabolites reported in this study) were 
TABLE 4

Summary of estimated parameter values for glyburide and metabolites in the SCHH model (mean and 95\% CI)

\begin{tabular}{|c|c|c|c|c|c|}
\hline & $C L_{u, a c t}$ & $C L_{\text {u,pass }}$ & $C L_{u, \text { bile }}$ & $C L_{u, e f f l u x}$ & $f_{u, c}$ \\
\hline Glyburide & $11.8(9.0-16)$ & $13.8(9.3-15)$ & $\begin{aligned} \mu l \cdot \min ^{-1} \cdot \mathrm{mg} \text { protein }^{-1} \\
1.9\left(1.4 \times 10^{-5} \text { to } 3.6\right)\end{aligned}$ & $5.5 \times 10^{-5}\left(7.9 \times 10^{-7}\right.$ to 1.7$)$ & $0.0301^{a}$ (fixed) \\
\hline M1 & $3.61(2.4-7.2)$ & $0.661^{b}(0.33-2.9)$ & $0.222\left(1.9 \times 10^{-9}\right.$ to 0.71$)$ & $0.634\left(1.9 \times 10^{-8}\right.$ to 3.1$)$ & $0.579^{a, b}$ (fixed) \\
\hline $\mathrm{M} 2 \mathrm{~b}$ & $7.20(4.6-11)$ & & $0.0866\left(6.7 \times 10^{-8}\right.$ to 1.5$)$ & $1.14\left(4.7 \times 10^{-9}\right.$ to 2.7$)$ & \\
\hline
\end{tabular}

${ }^{a} f_{u, c}$ was estimated from PHHs after longer incubations (Supplemental Fig. 1).

${ }^{b}$ Assumed to be the same for both metabolites to minimize overparameterization of the mechanistic model used to estimate in vitro transport kinetics in hepatocyte systems.

generated after combining priors from step 1 and likelihood from step 2 by using an adaptive Markov chain Monte Carlo (MCMC) approach. The adaptive MCMC has been published previously (Haario et al., 2006) and implemented in the MCMC toolbox for MATLAB (http://helios.fmi.fi/ lainema/mcmc/\#sec-4). The starting position of MCMC chains and initial error variance were set with the globally optimized values.

\section{Results}

Substrate Affinity of Glyburide and Metabolites to Hepatic and Renal Transporters. Glyburide is transported by OATP1B1, with the uptake by HEK293 cells transfected with OATP1B1 being significantly higher $(P<0.05)$ than by HEK-mock cells (Table 1$)$. The uptake ratio at $10 \mu \mathrm{M}$ is lower than that at $1 \mu \mathrm{M}$, indicating saturation at a higher concentration, which is consistent with our previous results (MichaelisMenten constant, $2 \mu \mathrm{M}$ ) (Varma et al., 2014). Our previous studies (Varma et al., 2014) suggested that glyburide is not transported by OATP1B3 or OATP2B1. Metabolites M1 and M2b showed substrate affinity to all three hepatic OATP isoforms with uptake ratios generally over 20. Both metabolites were identified as substrates of the canalicular efflux transporters BCRP and MDR1, although neither metabolite showed affinity to MRP2 (Table 2). M1 was also a substrate of the basolateral efflux transporters MRP3 and MRP4, with the transport into the membrane vesicles being significantly higher $(P<0.05)$ in the presence of ATP than in the presence of AMP. M2b, on the other hand, was not transported by MRP3 but showed significant transport by MRP4 at $10 \mu \mathrm{M}$ substrate concentration. Neither parent nor the metabolites showed substrate affinity to renal OAT1; however, both metabolites were transported by OAT3 (Table 3).

Hepatic Disposition of Glyburide and Metabolites Using Human Hepatocyte Assays. Primary human hepatocytes in plated culture were used to assess the involvement of active uptake in the hepatic disposition and to determine the hepatobiliary transport kinetics (Fig. 1; Supplemental Fig. 1). Rifamycin SV significantly inhibited the hepatic uptake of glyburide, M1, and M2b in both $\mathrm{PHH}$ and SCHH studies. SCHH data were simultaneously fitted to estimate in vitro transport parameters using mathematical models (Table 4). While statistically significant active uptake was discerned for the three compounds, due to limited data points during the efflux phase estimated basal efflux and biliary excretion were associated with large uncertainties. The metabolic stabilities of M1 and M2b were assessed using HLM and suspension human hepatocytes, wherein, neither metabolite showed any measureable turnover in HLM incubations (up to 1 hour) with and without NADPH and only $\sim 15-$ $30 \%$ depletion at the end of 5 hours of incubation in human hepatocytes. $\mathrm{M} 1$ and M2b did not convert to each other or to the parent in these studies. Based on these findings, we assumed further metabolism of M1 and M2b to be negligible for PBPK modeling and simulations.

Pharmacokinetic Characterization of Glyburide and Metabolites. The whole-body PBPK model implementing the multiple hepatic transport processes and observed renal clearance $\left(\mathrm{CL}_{\mathrm{R}}\right)$ reasonably described clinical pharmacokinetics of glyburide and its metabolites (Fig. 2). By combining information from in vitro SCHH data and IVIVE knowledge from other compounds, the Bayesian inference could provide reasonably confident parameter estimates (Supplemental Fig. 3; Table 5). Due to a lack of prior IVIVE knowledge, $C L_{\text {liver, }, \text { bile, } M}$ and $C L_{\text {liver }, u, \text { efflux }, M}$ were obtained purely from fitting the clinical data. For both $C L_{\text {liver,u,bile,M }}$ and $C L_{\text {liver,u,efflux,M, a value greater than } 101 / \text { hour }}$ seemed to be necessary to describe the clinical data. To show the importance of prior information in decreasing estimation uncertainty, the estimated (posterior) parameter distributions with and without prior information are provided in Supplemental Figs. 3 and 4. To understand the empirical IVIVE scaling factors required to bridge in vitro data and in vivo data in this study, we calculated the following ratios of posterior mean of hepatic clearance processes to physiologically scaled in vitro clearances: 44.5 for active uptake of glyburide; 0.760 for passive diffusion of glyburide; and 0.192 for metabolism of glyburide; 30.3 for active uptake of metabolites; and 0.0860 for passive diffusion of metabolites. With the posterior distributions of fitted parameters, we simulated liver concentrations of glyburide, M1, and M2b. The predicted pseudo-steady-state unbound liver-to-unbound plasma ratios $\left(K p_{u u}\right)$ were about 32 (95\% CI, 15-53) and 3.7 (95\% CI, 0.092-39) for glyburide and metabolites, respectively.

Inhibition of BSEP and NTCP by Glyburide and Metabolites. The uptake of taurocholic acid by human BSEP and NTCP was inhibited in the presence of glyburide and metabolites in a dose-dependent manner (Fig. 3). Glyburide was a more potent inhibitor against both BSEP and NTCP than its metabolites. Interestingly, although the two metabolites showed similar inhibition potencies $\left(\mathrm{IC}_{50}\right)$ against BSEP, M1 was less potent than M2b against NTCP.

Predicted Inhibition of BSEP and NTCP in Human Liver. Based on the PBPK model and the posterior distributions of fitted parameters, we prospectively simulated the liver intracellular free concentrations of glyburide and metabolites after oral dosing of glyburide $10 \mathrm{mg} /$ day for 3 days. Assuming that the inhibition follows a free concentration-direct response model [i.e., $C_{\text {liver,free }} /\left(C_{\text {liver,free }}+\mathrm{IC}_{50}\right)$ ], we simulated the BSEP and NTCP inhibition in vivo for the parent and metabolites using mean $\mathrm{IC}_{50}$ values determined in the in vitro assay. The simulations were performed individually for each compound without considering the interactions among inhibitors. The simulation showed that the three compounds may cause only minimal inhibition of BSEP and NTCP in vivo ( $<10 \%$ inhibition) (Fig. 4, A-D). In addition, we simulated the inhibition based on total liver concentrations, where only glyburide showed a stronger inhibition-up to 25\% BSEP inhibition and $75 \%$ NTCP inhibition (Fig. 4, E-H).

\section{Discussion}

Collective data from this study depict that glyburide is primarily cleared from the blood compartment by hepatic uptake via OATP1B1 and subsequently metabolized, whereas the hepatic disposition of its 

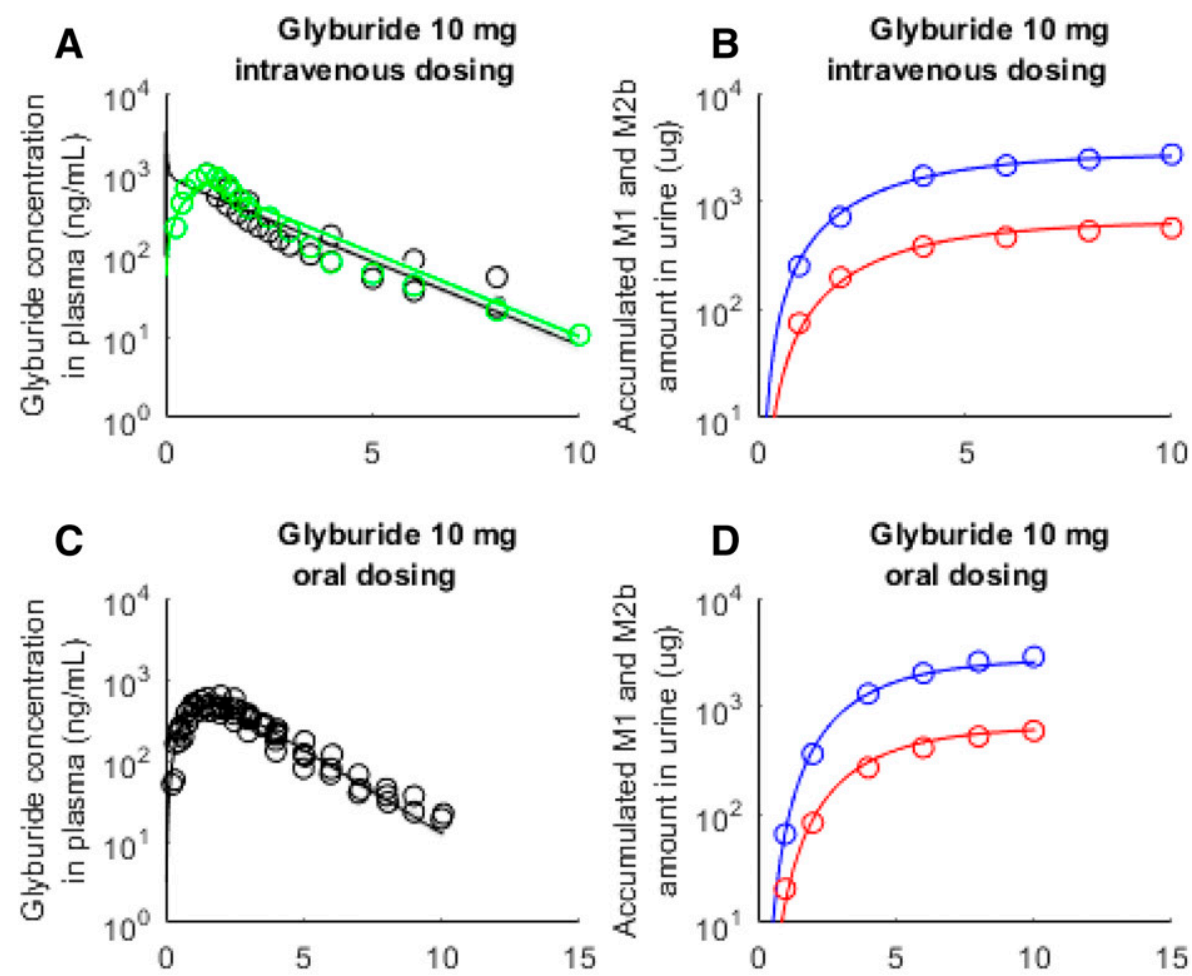

Fig. 2. Observed (circles) and simulated (lines) plasma concentration-time profiles and urinary recoveries of glyburide (black), M1 (blue), and M2b (red) after intravenous dosing of glyburide (A and B), oral dosing

E

M1 $10 \mathrm{mg}$
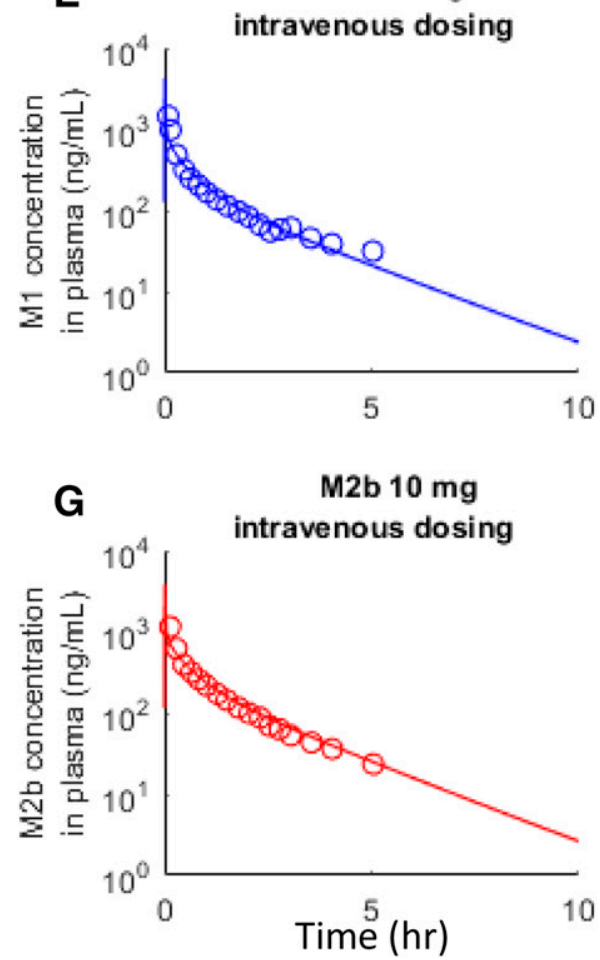
of glyburide (C and D), intravenous dosing of M1 (E and $\mathrm{F}$ ), and intravenous dosing of $\mathrm{M} 2 \mathrm{~b}(\mathrm{G}$ and $\mathrm{H})$.

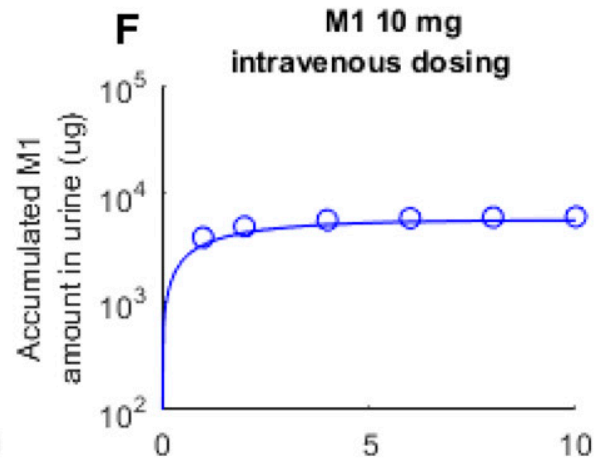

Green and black in (A) indicate infusion and bolus dosing, respectively.



active hydroxyl metabolites, M1 and M2b, are determined by hepatic uptake transporters (OATP1B1, OATP1B3 and OATP2B1) and biliary [BCRP and P-glycoprotein (P-gp)] and basolateral (MRP3 and MRP4) efflux pumps (Fig. 5). Additionally, the metabolites are substrates to the renal transporter OAT3, which is likely mediating their significant active secretion (observed human $C L_{R} / f_{u, p}$. GFR is $>25$ ) into urine. These two metabolites of glyburide possess considerable hypoglycemic activity at their clinically relevant plasma concentrations. After single intravenous dosing of glyburide, M1, and M2b, separately, blood glucose and serum insulin levels are significantly changed by the parent as well as the two metabolites (Rydberg et al., 1994). At an oral dose of glyburide of about $10 \mathrm{mg}$, metabolite levels are higher than those of glyburide, with high metabolite levels found at least 10-16 hours after glyburide intake (Jonsson et al., 2001) in patients with type 2 diabetes. This implies that 
TABLE 5

Summary of PBPK model parameter estimates and their 95\% CIs of glyburide and metabolites

\begin{tabular}{|c|c|c|c|c|}
\hline \multirow[t]{2}{*}{ Parameter } & \multirow[t]{2}{*}{ Unit } & \multicolumn{3}{|c|}{ Value } \\
\hline & & Glyburide & M1 & M2b \\
\hline$p K a^{a}$ & & 5.38 & 5.08 & 5.08 \\
\hline $\log \mathrm{D}_{7.4}{ }^{a}$ & & 2.1 & -0.48 & -0.43 \\
\hline$f_{u, p}$ & & $0.0028(0.0026-0.0030)$ & $0.034(0.032-0.036)$ & $0.024(0.023-0.025)$ \\
\hline$R_{B / P}$ & & 0.571 & $0.437(0.42-0.45)$ & $0.493(0.45-0.54)$ \\
\hline$F_{a} F_{g}{ }^{b}$ & & 1 & 0.5 & 0.5 \\
\hline$k_{a}^{c, d^{8}}$ & Hour & $1.85(1.64-2.08)$ & 0.5 & 0.5 \\
\hline$C L_{R}^{d, e}$ & $1 /$ hour & 0 & 7.18 & 7.18 \\
\hline$C L_{\text {liver,u,pass }}{ }^{d}$ & $1 /$ hour & $111(24.9-345)$ & $0.602(0.136-11.2)$ & $0.602(0.136-11.2)$ \\
\hline$C L_{\text {liver,u,act }}$ & $1 /$ hour & $5560(2400-11,700)$ & $1730(723-6620)$ & $1730(723-6620)$ \\
\hline$C L_{\text {liver, } u, \text { met }}{ }$ & $1 /$ hour & $71.9(47.3-159)$ & 0 & 0 \\
\hline$C L_{\text {liver }, \text {, }, \text { bile }} d, f$ & $1 /$ hour & 0 & $104(10.7-4210)$ & $104(10.7-4210)$ \\
\hline$C L_{\text {liver }, u, \text { efflux }} d, f$ & 1/hour & 0 & $508(28.5-12,000)$ & $508(28.5-12,000)$ \\
\hline Metabolite $F_{M I}^{d}$ & & & $0.564(0.490-0.690)$ & \\
\hline Metabolite $F_{M 2 b^{g}}$ & & & & 0.113 \\
\hline $\mathrm{IC}_{50, \mathrm{BSEP}}$ & $\mu \mathrm{M}$ & $7.45(6.58-8.44)$ & $34.9(27.1-44.9)$ & $36.7(29.2-46.2)$ \\
\hline $\mathrm{IC}_{50, \mathrm{NTCP}}$ & $\mu \mathrm{M}$ & $0.5(0.37-0.66)$ & $771(314-1892)$ & $8.1(5.6-11.8)$ \\
\hline
\end{tabular}

$C L_{\text {liver, }, \text {,met }}$, hepatic unbound metabolism clearance; $k_{a}$, first-order absorption rate; $R_{B / P}$, blood-to-plasma ratio.

${ }_{\mathrm{pKa}}$ and $\log \mathrm{D}_{7.4}$ values are generated in-house for glyburide and are predicted using ACD/Labs (Toronto, Ontario, Canada) for metabolites.

${ }^{b}$ The fraction absorbed and escaped from intestinal metabolism $\left(F_{a} F_{g}\right)$ is fixed at 1 for glyburide based on clinical observations. Due to lack of data, The $F_{a}$ for metabolites is fixed at 0.5 considering their low-to-moderate passive permeability, whereas $F_{g}$ is assumed to be for their low metabolism.

${ }^{c}$ The absorption rate of metabolites is fixed at 0.5 arbitrarily due to limited data. This involves reabsorption of metabolites from the intestine after biliary secretion (enterohepatic recycling).

${ }^{d}$ The $95 \%$ CIs are approximated as 2.5 and 97.5 percentiles of values generated in Bayesian inference. M1 and M2b are assumed to share the same hepatic uptake characteristics as indicated in the text.

${ }^{e}$ The $C L_{R}$ is directly determined from the ratio of amount of urinary execration and plasma exposure. As such, its variability is more of the individual variability (not addressed here) than numerical uncertainty.

${ }^{f} C L_{\text {liver,u,bile }}$ and $C L_{\text {liver,u,efflux }}$ are correlated by fitting clinical data, whereas there is no prior information about these two parameters, as such, and the large uncertainty associated with them.

${ }^{g} F_{M 2 b}$ is fixed as one-fifth of $F_{M 1}$ based on clinical observations. About 25-30\% and 5-6\% of M1 and M2b, respectively, are recovered in the urine after intravenous glyburide or oral dosing, whereas equal mounts $(60 \%)$ of $\mathrm{M} 1$ and M2b are recovered in the urine after intravenous M1 and M2b dosing (Rydberg et al., 1995).

the metabolites contribute to the hypoglycemic effect with a longer effect duration than the parent itself and that they may be leading to the long-lasting hypoglycemic events noted with glyburide (Asplund et al., 1983; Rydberg et al., 1997). Overall, this study demonstrates for the first time an important role for hepatic and renal transporters in the pharmacokinetics of glyburide-active metabolites, suggesting that the functional changes in these processes due to age, sex, disease, genetic variation, or DDIs could significantly alter the plasma exposure of the metabolites and consequently modulate hypoglycemic activity, which may be of clinical importance.

Mathematical modeling was employed for SCHH data to evaluate hepatocyte vectorial transport and further estimate the intrinsic transport rates to execute mechanistic PBPK modeling. SCHH as well as 75-minute plated hepatocyte studies suggested significant active uptake for all three compounds, which is associated with OATP substrate activity, as demonstrated using transporter-transfected cells. However,
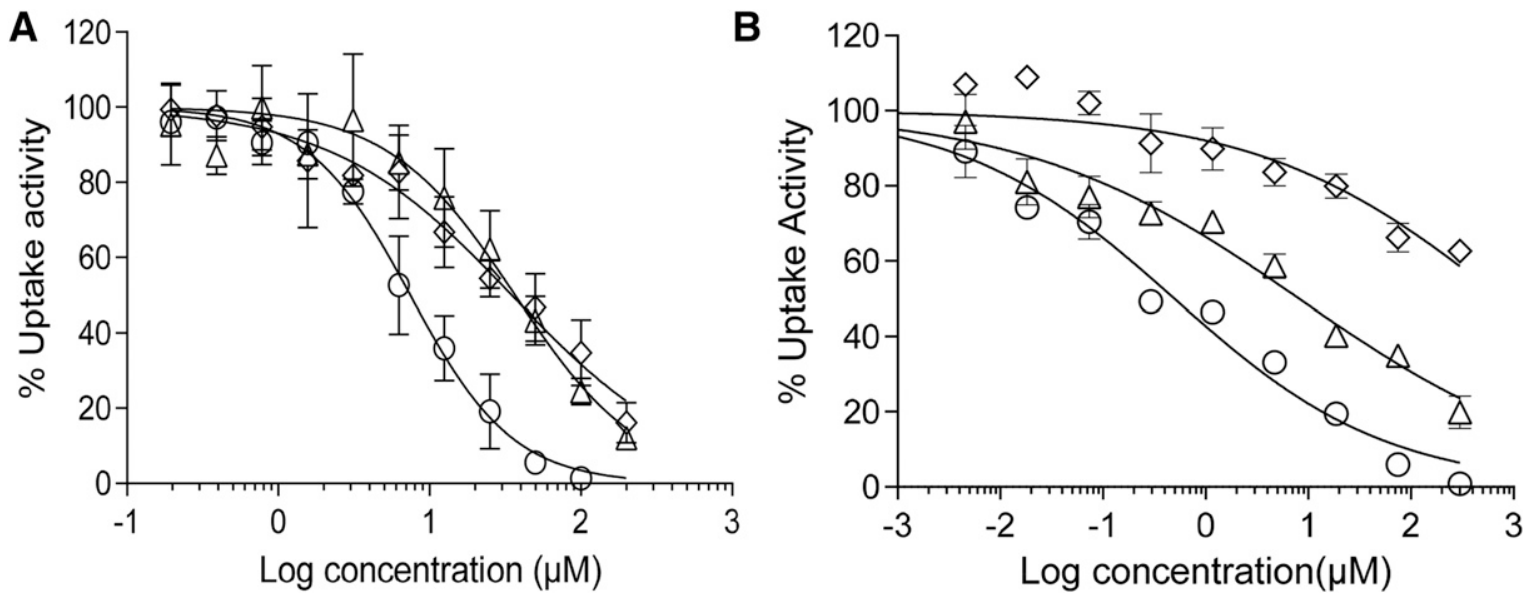

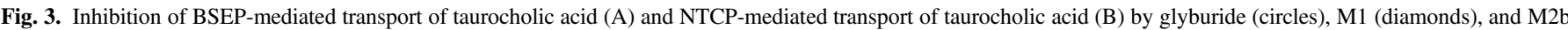

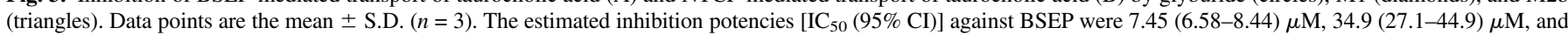

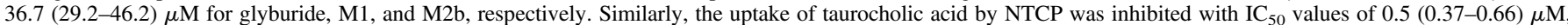
771 (314-1892) $\mu \mathrm{M}$, and 8.1 (5.6-11.8) $\mu \mathrm{M}$ by glyburide, M1, and M2b, respectively. 

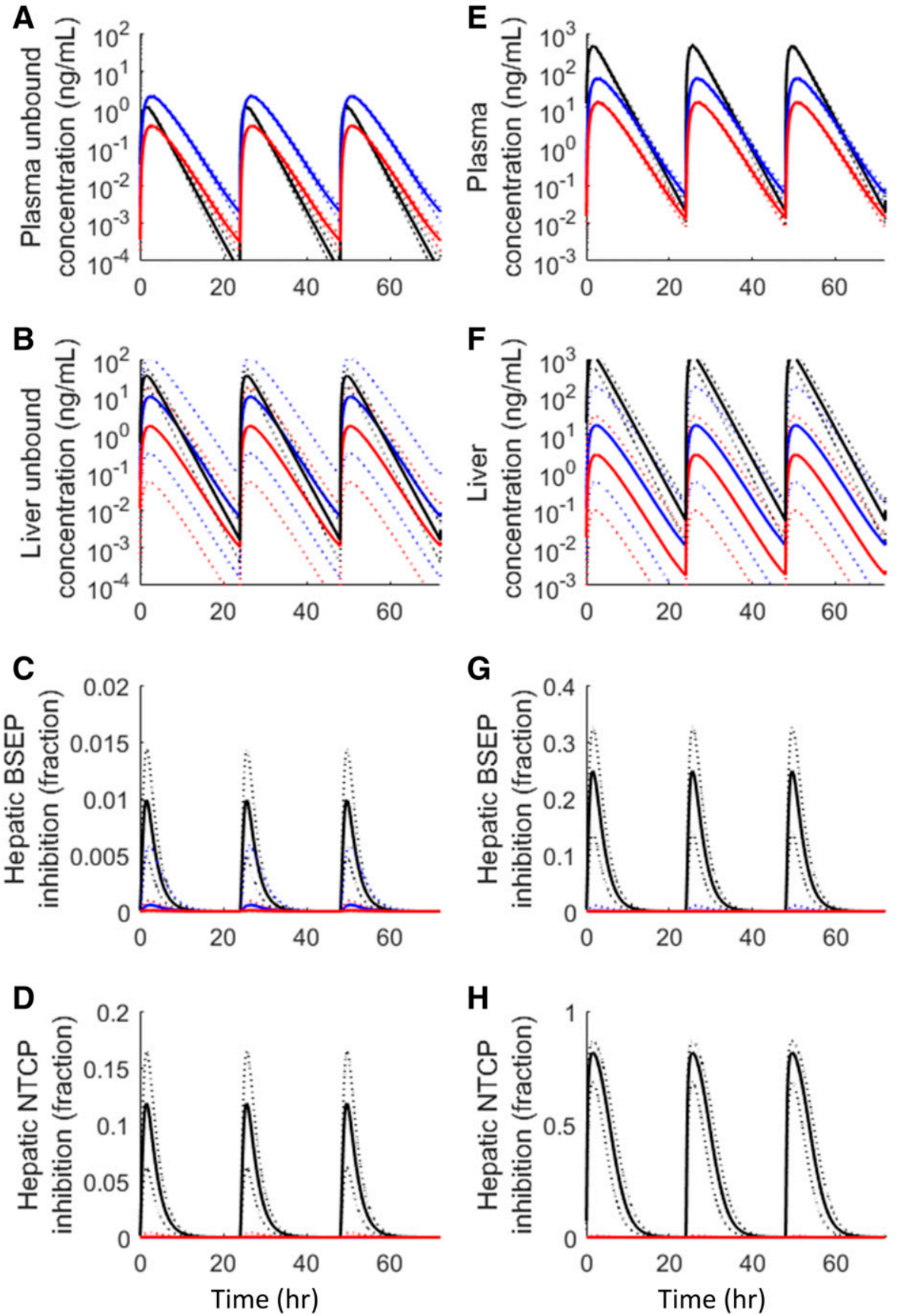

Fig. 4. Predicted plasma (A and $\mathrm{E}$ ) and liver tissue concentrations (B and F) of glyburide (black), M1 (blue), and M2b (red) and the fraction of inhibited BSEP (C and G) and NTCP (D and H) after 10-mg glyburide oral dosing per day for 3 days. Plots (A-D) represents unbound concentrations and inhibition based on unbound concentrations. Plots $(\mathrm{E}-\mathrm{H})$ represent total concentrations and inhibition based on total concentrations. The solid and dotted lines represent median predictions and 2.5 and 97.5 percentiles.
SCHH could not discern statistically significant basolateral efflux or biliary clearance for any compound, leading to uncertain estimates for both parameters, although membrane vesicle studies suggested that metabolites are transported by BCRP and P-gp (biliary) and MRP3 and/ or MRP4 (basolateral) efflux pumps (Tables 2 and 4). This may be attributed to limited sensitivity for these possibly slow efflux processes and the experimental variability in the $\mathrm{SCHH}$ system. However, the pharmacokinetics of the metabolites were best described by the PBPK model, with the estimated active basolateral efflux clearance (i.e., total efflux minus passive and biliary clearances) higher than the biliary clearance, implying that metabolites are preferentially pumped into blood (Table 5). These hydrophilic metabolites, which likely are formed in the liver after glyburide dosing, are primarily eliminated by the kidneys, supporting the importance of basolateral efflux in their hepatic handling (Rydberg et al., 1995). Evidently, the interplay of uptake and biliary and basolateral efflux transporters are key determinants of the pharmacokinetics of these metabolites.

Predicting or evaluating the pharmacokinetics of metabolites is challenging, particularly when their disposition involves membrane transporters (Zamek-Gliszczynski et al., 2014; Kimoto et al., 2015; Templeton et al., 2016). Here, we developed a PBPK model considering hepatobiliary transport and metabolism to characterize the pharmacokinetics of glyburide and its metabolites. Our group previously estimated system-specific empirical scaling factors for hepatic active and passive transport and metabolism by simultaneously fitting clinical observations of seven compounds with global optimization methods (Li et al., 2014b). We employed the same mechanistic model combined with a Bayesian approach, which naturally combines in vitro data, IVIVE scaling factors 


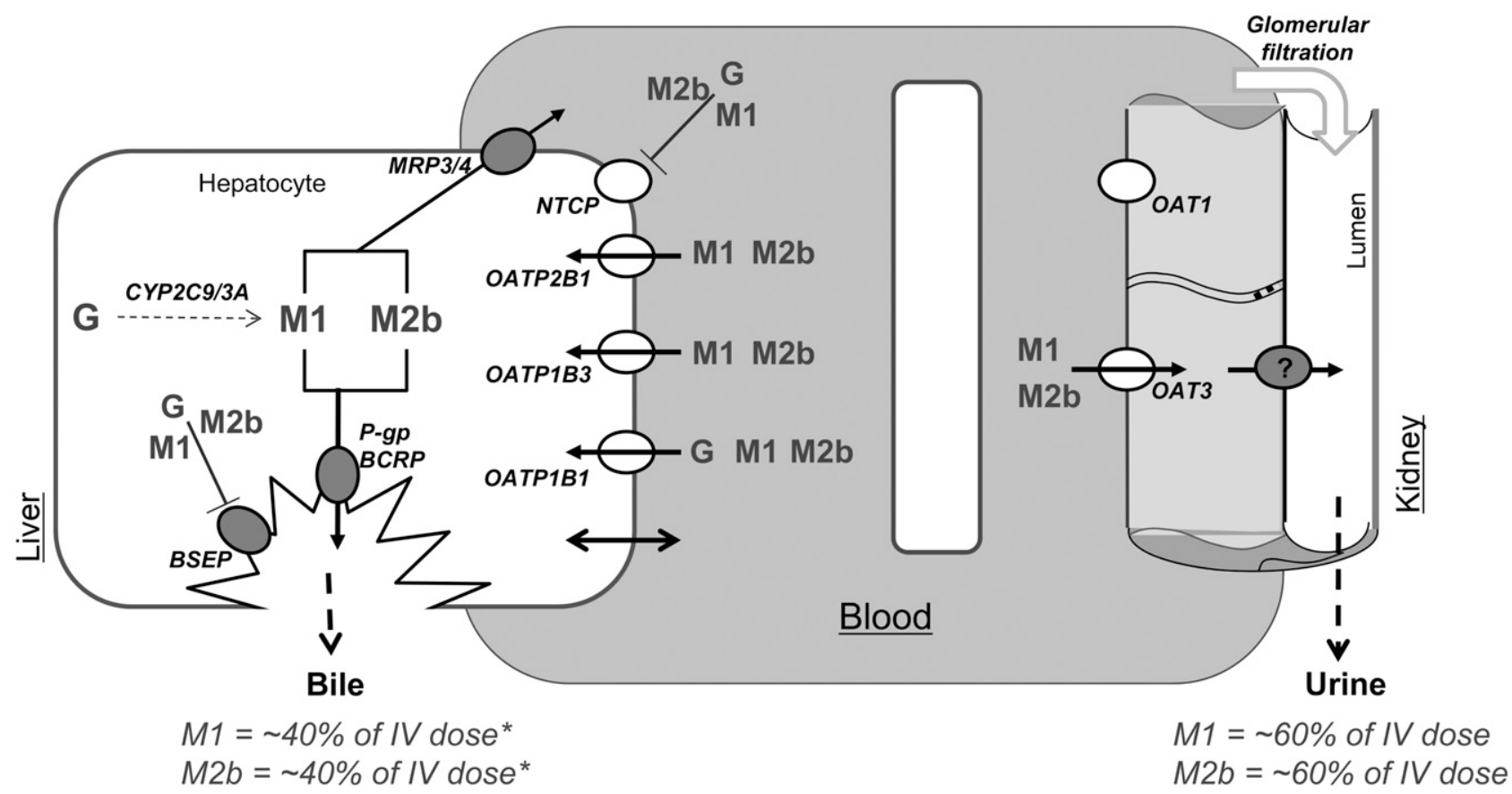

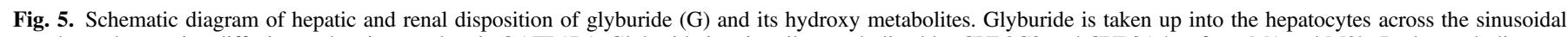

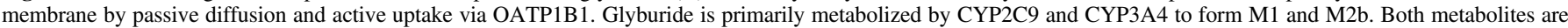

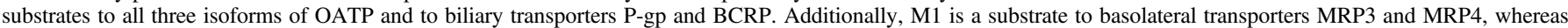

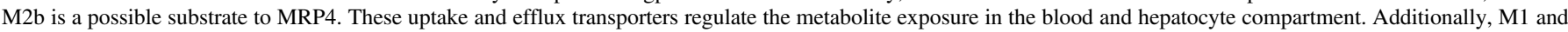

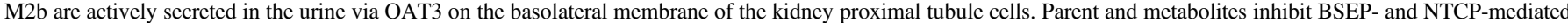

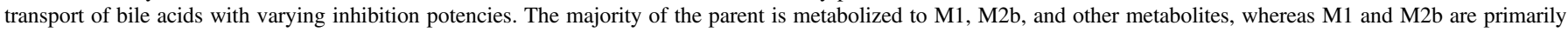
excreted in the urine and bile.

determined previously (prior knowledge), and the clinical data (likelihood), to characterize the plasma pharmacokinetics of parent and metabolites and to further effectively decrease the uncertainty in the parameter estimations and liver concentration predictions. The model described the plasma concentration-time profiles of the parent and metabolites and recovered the renal excretion profiles reasonably well (Fig. 3). On the basis of the PBPK model simulations, the following mechanistic information can be derived. First, hepatic uptake transporters play a predominant role in the systemic clearance of glyburide and metabolites. Second, due to higher plasma protein binding of the parent, our PBPK model simulations suggested comparable unbound plasma concentrations and a higher unbound trough concentration of metabolites, particularly of M1, compared with glyburide (Fig. 4A). These findings signify the potential contribution of metabolites to the pharmacodynamic activity and, additionally, their larger role in the long-lasting hypoglycemic effects of glyburide (Asplund et al., 1983; Rydberg et al., 1997; Jonsson et al., 2001).

The role of transporter-enzyme interplay in the hepatic clearance of glyburide can be corroborated by its clinical DDIs. For instance, coadministration of a single intravenous dose of rifampicin (OATP inhibitor) increases the plasma area under the curve of glyburide and consequently increases hypoglycemic effects, whereas rifampicin multiple-dose oral treatment (cytochrome P450 induction and OATP inhibition) shows minimal impact on glyburide exposure and pharmacodynamics (Zheng et al., 2009). Using a similar PBPK approach, we previously rationalized the magnitude of change in glyburide pharmacokinetics caused by several cytochrome P450 inhibitors/inducers and/ or OATP inhibitors (Varma et al., 2014). The current study suggests that the reduced functional activities of hepatic OATPs and/or renal OAT3 associated with DDIs, age, and genetic variation could increase, whereas those of MRP3/4 may decrease systemic exposure of M1 and M2b, leading to variability in pharmacodynamic response.
Glyburide is a relatively safe drug; however, it has been implicated in occasional cases of cholestatic jaundice and hepatocellular disease and in a few cases of granulomatous hepatitis (van Basten et al., 1992; Krivoy et al., 1996; Saw et al., 1996). Additionally, the bosentan-glyburide combination is contraindicated as emphasized with a black box warning on the bosentan product label. Here, we showed that glyburide major metabolites inhibit BSEP in vitro, although the inhibition potencies were about 5-fold lower compared with the parent (Fig. 3). We further evaluated the potential for glyburide and metabolites to inhibit BSEP in vivo considering the concentration-response effect on the basis of in vitro inhibition potencies $\left(\mathrm{IC}_{50}\right)$, and the PBPK model simulated unbound hepatic concentrations (Fig. 4). Although the free liver concentrations were projected to be severalfold ( $\sim 40$ times) higher than plasma free concentrations, glyburide and metabolites could not produce significant inhibition of BSEP, which implies that 1) there is a potential disconnect between in vitro and in vivo inhibition potencies and/or 2) BSEP inhibition is not the major cause of glyburide-induced cholestasis, with other mechanisms potentially involved. Generally, BSEP inhibition is one of numerous potential mechanisms leading to drug-induced cholestasis, and evaluation of this liability in isolation may not provide an overall assessment of toxicity (Rodrigues et al., 2014; Shon and Abernethy, 2014). Alternatively, Woodhead et al. (2014) suggested a relationship between maximum plasma glyburide concentration and the change in bile acids exposure using DILIsym, a mechanistic model of druginduced liver injury (Woodhead et al., 2014). We therefore evaluated in vivo BSEP inhibition assuming total hepatic concentrationresponse effect. Under this assumption, the model predicted up to $25 \%$ BSEP inhibition by glyburide, whereas the metabolites showed no notable inhibition (Fig. 4C). Moreover, considering the free or 
total concentration-response effect, glyburide, but not the metabolites, showed notable inhibition of NTCP, which plays a key role in the hepatic uptake and regulates systemic exposure of bile acids.

The $K p_{u u}$ of glyburide was predicted to be $\sim 32(95 \% \mathrm{CI}=15-53)$. However, the predicted metabolite $K p_{u и}$ was low ( $\left.\sim 3.7\right)$ and with larger variability due to uncertainty in biliary and basolateral efflux parameter estimates. Although it is not easy to verify the human liver exposure predictions due to limitations in obtaining appropriate clinical data (i.e., in vivo liver concentration of parent and metabolites), our rationale for using the current PBPK approach comes from a previous study (Li et al., 2016) demonstrating an accurate (but not necessarily precise) prediction of liver concentrations when fitting plasma profile data. Additionally, a Bayesian approach was applied to improve the precision in this study. However, in Bayesian we cannot rule out the possibility that our prior IVIVE knowledge is biased, resulting in the underprediction of liver exposure. As such, we simulated liver exposure, BSEP, and NTCP inhibition again after removing priors from MCMC. Although the new prediction bands are wider (i.e., more likely to cover real exposure) (Supplemental Fig. 4), the conclusion of minimal inhibition on NTCP and BSEP has not changed (Supplemental Fig. 5). One could argue whether a different model structure or optimization process could conclude a much more significant inhibition of BESP. Based on simple calculations, we note that, if a $10-\mathrm{mg}$ glyburide dose was injected directly into a 1.25-1 liver without considering transport or metabolism, the unbound glyburide concentration would be $\sim 0.5 \mu \mathrm{M}$, leading only to about a $6 \%$ competitive inhibition given current $\mathrm{IC}_{50}$ values $(\sim 7.5 \mu \mathrm{M})$. The study did not investigate transinhibition of BSEP by the metabolites in the bile because of challenges in simulating physiologically relevant concentrations of metabolites in the bile. However, given that a significant amount of metabolites is excreted into the bile, their concentrations in bile could be higher than their concentrations in plasma and liver. Alternatively, glyburide and metabolites may have a cooperative effect leading to more severe BSEP inhibition in vivo. Further understanding in the area concerning disconnect in exposure-response effect with the free drug hypothesis is warranted to rationalize the role of BSEP inhibition in glyburide-induced cholestasis.

In conclusion, we characterized the mechanisms involved in the disposition and pharmacokinetics of glyburide as well as its pharmacologically active metabolites. Glyburide is actively taken up by hepatocytes via OATP1B1, whereas both metabolites were identified as substrates of multiple hepatic and renal transporters. A PBPK model with Bayesian analysis verified the clinical relevance of these multiple transporter processes in determining systemic and tissue exposure of parent and metabolites with implications for the pharmacodynamic drug response. Finally, this approach can be applied to other drugmetabolite pairs to predict or better characterize their pharmacokinetics/ pharmacodynamics.

\section{Acknowledgments}

We thank Tristan Maurer, Hugh Barton, David Rodrigues, and Larry Tremaine for valuable inputs during this work.

\section{Authorship Contributions}

Participated in research design: $\mathrm{Li}, \mathrm{Bi}$, Vildhede, Scialis and Varma.

Conducted experiments: Bi, Vildhede, Scialis, Yang, Marroquin, and Lin.

Contributed new reagents or analytic tools: $\mathrm{Li}$.

Performed data analysis: Li, Bi, Vildhede, Scialis, and Varma.

Wrote or contributed to the writing of the manuscript: $\mathrm{Li}, \mathrm{Bi}$, Vildhede, Scialis, Mathialagan, Yang, Marroquin, Lin, and Varma.

\section{References}

Asplund K, Wiholm BE, and Lithner F (1983) Glibenclamide-associated hypoglycaemia: a report on 57 cases. Diabetologia 24:412-417.

Bi YA, Kazolias D, and Duignan DB (2006) Use of cryopreserved human hepatocytes in sandwich culture to measure hepatobiliary transport. Drug Metab Dispos 34:1658-1665.

Chalk JB, Patterson M, Smith MT, and Eadie MJ (1986) Correlations between in vitro dissolution, in vivo bioavailability and hypoglycaemic effect of oral glibenclamide. Eur J Clin Pharmacol 31:177-182.

Dawson S, Stahl S, Paul N, Barber J, and Kenna JG (2012) In vitro inhibition of the bile salt export pump correlates with risk of cholestatic drug-induced liver injury in humans. Drug Metab Dispos 40:130-138.

Fattinger K, Funk C, Pantze M, Weber C, Reichen J, Stieger B, and Meier PJ (2001) The endothelin antagonist bosentan inhibits the canalicular bile salt export pump: a potential mechanism for hepatic adverse reactions. Clin Pharmacol Ther 69:223-231.

Feldman JM (1985) Glyburide: a second-generation sulfonylurea hypoglycemic agent. History, chemistry, metabolism, pharmacokinetics, clinical use and adverse effects. Pharmacotherapy $\mathbf{5}$ : 43-62.

Haario H, Laine M, Mira A, and Saksman E (2006) DRAM: efficient adaptive MCMC. Stat Comput 16:339-354.

Jönsson A, Hallengren B, Rydberg T, and Melander A (2001) Effects and serum levels of glibenclamide and its active metabolites in patients with type 2 diabetes. Diabetes Obes Metab 3: $403-409$.

Kimoto E, Li R, Scialis RJ, Lai Y, and Varma MV (2015) Hepatic disposition of gemfibrozil and its major metabolite gemfibrozil 1-O- $\beta$-glucuronide. Mol Pharm 12:3943-3952.

Kirchheiner J, Brockmöller J, Meineke I, Bauer S, Rohde W, Meisel C, and Roots I (2002) Impact of CYP2C9 amino acid polymorphisms on glyburide kinetics and on the insulin and glucose response in healthy volunteers. Clin Pharmacol Ther 71:286-296.

Krivoy N, Zaher A, Yaacov B, and Alroy G (1996) Fatal toxic intrahepatic cholestasis secondary to glibenclamide. Diabetes Care 19:385-386.

Li R, Barton HA, and Maurer TS (2014a) Toward prospective prediction of pharmacokinetics in OATP1B1 genetic variant populations. CPT Pharmacometrics Syst Pharmacol 3:e151.

Li R, Barton HA, Yates PD, Ghosh A, Wolford AC, Riccardi KA, and Maurer TS (2014b) A "middle-out" approach to human pharmacokinetic predictions for OATP substrates using physiologically-based pharmacokinetic modeling. J Pharmacokinet Pharmacodyn $\mathbf{4 1}$ 197-209.

Li R, Maurer TS, Sweeney K, and Barton HA (2016) Does the systemic plasma profile inform the liver profile? Analysis using a physiologically based pharmacokinetic model and individual compounds. AAPS $J$ 18:746-756.

Lilja JJ, Niemi M, Fredrikson H, and Neuvonen PJ (2007) Effects of clarithromycin and grapefruit juice on the pharmacokinetics of glibenclamide. Br J Clin Pharmacol 63:732-740.

Mylona P and Cleland JG (1999) Update of REACH-1 and MERIT-HF clinical trials in heart failure. Cardio.net Editorial Team. Eur J Heart Fail 1:197-200.

Naritomi Y, Terashita S, and Kagayama A (2004) Identification and relative contributions of human cytochrome P450 isoforms involved in the metabolism of glibenclamide and lansoprazole: evaluation of an approach based on the in vitro substrate disappearance rate. Xenobiotica 34:415-427.

Neugebauer G, Betzien G, Hrstka V, Kaufmann B, von Möllendorff E, and Abshagen U (1985) Absolute bioavailability and bioequivalence of glibenclamide (Semi-Euglucon N). Int J Clin Pharmacol Ther Toxicol 23:453-460.

Niemi M, Backman JT, Neuvonen M, Neuvonen PJ, and Kivistö KT (2001) Effects of rifampin on the pharmacokinetics and pharmacodynamics of glyburide and glipizide. Clin Pharmacol Ther 69:400-406.

Niemi M, Cascorbi I, Timm R, Kroemer HK, Neuvonen PJ, and Kivistö KT (2002) Glyburide and glimepiride pharmacokinetics in subjects with different CYP2C9 genotypes. Clin Pharmacol Ther 72:326-332.

Ravindran S, Zharikova OL, Hill RA, Nanovskaya TN, Hankins GD, and Ahmed MS (2006) Identification of glyburide metabolites formed by hepatic and placental microsomes of humans and baboons. Biochem Pharmacol 72:1730-1737.

Rodgers T and Rowland M (2006) Physiologically based pharmacokinetic modelling 2: predicting the tissue distribution of acids, very weak bases, neutrals and zwitterions. J Pharm Sci 95: $1238-1257$.

Rodrigues AD, Lai Y, Cvijic ME, Elkin LL, Zvyaga T, and Soars MG (2014) Drug-induced perturbations of the bile acid pool, cholestasis, and hepatotoxicity: mechanistic considerations beyond the direct inhibition of the bile salt export pump. Drug Metab Dispos $\mathbf{4 2}$ 566-574.

Rydberg T, Jönsson A, Karlsson MO, and Melander A (1997) Concentration-effect relations of glibenclamide and its active metabolites in man: modelling of pharmacokinetics and pharmacodynamics. Br J Clin Pharmacol 43:373-381.

Rydberg T, Jönsson A, and Melander A (1995) Comparison of the kinetics of glyburide and its active metabolites in humans. $J$ Clin Pharm Ther 20:283-295.

Rydberg T, Jönsson A, Røder M, and Melander A (1994) Hypoglycemic activity of glyburide (glibenclamide) metabolites in humans. Diabetes Care 17:1026-1030.

Saw D, Pitman E, Maung M, Savasatit P, Wasserman D, and Yeung CK (1996) Granulomatous hepatitis associated with glyburide. Dig Dis Sci 41:322-325.

Shon J and Abernethy DR (2014) Application of systems pharmacology to explore mechanisms of hepatotoxicity. Clin Pharmacol Ther 96:536-537.

Spraul M, Streeck A, Nieradzik M, and Berger M (1989) Uniform elimination pattern for glibenclamide in healthy Caucasian males. Arzneimittelforschung 39:1449-1450.

Templeton IE, Chen Y, Mao J, Lin J, Yu H, Peters S, Shebley M, and Varma MV (2016) Quantitative prediction of drug-drug interactions involving inhibitory metabolites in drug development: how can physiologically based pharmacokinetic modeling help? CPT Pharmacometrics Syst Pharmacol 5:505-515.

van Basten JP, van Hoek B, Zeijen R, and Stockbrügger R (1992) Glyburide-induced cholestatic hepatitis and liver failure. Case-report and review of the literature. Neth J Med 40:305-307.

Varma MV, Scialis RJ, Lin J, Bi YA, Rotter CJ, Goosen TC, and Yang X (2014) Mechanism-based pharmacokinetic modeling to evaluate transporter-enzyme interplay in drug interactions and pharmacogenetics of glyburide. AAPS $J$ 16:736-748.

Varma MV, Steyn SJ, Allerton C, and El-Kattan AF (2015) Predicting clearance mechanism in drug discovery: extended clearance classification system (ECCS). Pharm Res 32:3785-3802. 
Woodhead JL, Yang K, Brouwer KL, Siler SQ, Stahl SH, Ambroso JL, Baker D, Watkins PB, and Howell BA (2014) Mechanistic modeling reveals the critical knowledge gaps in bile acidmediated DILI. CPT Pharmacometrics Syst Pharmacol 3:e123.

Zamek-Gliszczynski MJ, Chu X, Polli JW, Paine MF, and Galetin A (2014) Understanding the transport properties of metabolites: case studies and considerations for drug development. Drug Metab Dispos 42:650-664.

Zharikova OL, Fokina VM, Nanovskaya TN, Hill RA, Mattison DR, Hankins GD, and Ahmed MS (2009) Identification of the major human hepatic and placental enzymes responsible for the biotransformation of glyburide. Biochem Pharmacol 78:1483-1490.

Zheng HX, Huang Y, Frassetto LA, and Benet LZ (2009) Elucidating rifampin's inducing and inhibiting effects on glyburide pharmacokinetics and blood glucose in healthy volunteers: unmasking the differential effects of enzyme induction and transporter inhibition for a drug and its primary metabolite. Clin Pharmacol Ther 85:78-85.

Zhou L, Naraharisetti SB, Liu L, Wang H, Lin YS, Isoherranen N, Unadkat JD, Hebert MF, and Mao Q (2010) Contributions of human cytochrome P450 enzymes to glyburide metabolism. Biopharm Drug Dispos 31:228-242.

Address correspondence to: Manthena V.S. Varma, Pharmacokinetics, Dynamics, and Metabolism, MS 8220-2451, Pfizer Global Research and Development, Pfizer Inc., Groton, CT 06340. E-mail: manthena.v.varma@pfizer.com 